\title{
Retos para la sostenibilidad en el Área metropolitana del Valle de Aburrá de Antioquia Colombia. Percepciones acerca de lo que entendemos por este término y ejemplos de actividades educativas
}

\author{
John Jairo Briceño Martínez ${ }^{*}$ y Equipo Área Educada ${ }^{1}$ \\ *Área Metropolitana del Valle de Aburrá. Calle 41 N 53-07. Medellin. Corporación Centro de Ciencia y \\ Tecnología de Antioquia. Carrera 46 número 56-11.Medellin..E-mail:johnjairob@hotmail.com
}

[Recibido en julio de 2011, aceptado en enero de 2012]

\begin{abstract}
Los retos de la sostenibilidad pasan por aspectos sociales, políticos, económicos, tecnológicos y educativos, que llevarán a desafiar cualquier programa a ir más allá de unas acciones puntuales para alcanzarla. El objetivo de este trabajo consistió en conocer la percepción de la sostenibilidad de los participantes durante las actividades desarrolladas por Área Educada, programa que promueve la sostenibilidad en los habitantes del Valle de Aburrá, Antioquia, Colombia. Se logró impactar 180.000 ciudadanos de los cuales damos cuenta de la percepción de 260 estudiantes y 100 profesores. Igualmente, se muestran dos ejemplos de cómo trabajar la sostenibilidad en el aula con profesores y estudiantes. Se concluye que los actores implicados en nuestras estrategias reconocen que la sostenibilidad tiene que ver con elementos físico naturales y de conservación de los recursos naturales, con una estrecha relación con el desarrollo económico por encima del desarrollo humano y social. No obstante, este último aspecto es altamente defendido por los estudiantes en comparación con los profesores.
\end{abstract}

Palabras clave: Sostenibilidad, Educación y Ambiente.

Challenges to the sustainability of Metropolitan Aburrá valley of Antioquia Colombia “Área Educada". Perceptions about what we understand for this term and examples of educational activities

The sustainability has to go through social, political, economic, technological and educational challenges that would take any program beyond a specific action to achieve it. The aim of this study was to examine the sustainability perception of the participants during the activities conducted by Área Educada, a metropolitan program of Valle de Aburrá, Antioquia Colombia. It managed to hit 180,000 citizens and made inquiries about the perception of 260 students and 100 teachers. Here is shown two examples of how to work sustainability into the classroom with teachers and students. We conclude that the stakeholders in our strategies recognize that sustainability is related to environmental elements and economic development. However, the human aspect is highly favored by the students compared with teachers.

Keywords: Sustainability, Educations and Environment.

\section{Introducción}

El Área metropolitana del Valle de Aburrá como autoridad en materia de planeación, transporte masivo y ambiente en Antioquia Colombia, 2009-2011, se propuso dentro de sus estrategias institucionales, asumir los retos de la sostenibilidad como un asunto fundamental en el desarrollo de la región. Como respuesta a este desafío territorial, creó el Programa Área Educada, una propuesta contundente para incidir directamente en los ciudadanos metropolitanos. Este trabajo se basa, en que un ciudadano educado y reflexivo, es un ciudadano responsable consigo mismo, con el ambiente y con la comunidad; por ello el Área Metropolitana invierte en ellos y ellas, en esos ciudadanos metropolitanos capaces de velar por la sostenibilidad a largo plazo.

\footnotetext{
${ }^{1}$ El autor desea agradecer a la entidad que desarrolla el Programa Área Educada: Área Metropolitana del Valle de Aburrá, cuyo director hasta el 2011 fue Mauricio Facio Lince Prada, a la gerencia del Programa realizada por el Centro de Ciencia y Tecnología de Antioquia en cabeza de Beatriz White Correa y al equipo coordinador: Área Educada, http://www.areaeducada.org/area/
} 
Área Educada busca mejorar la calidad educativa, construir infraestructuras sostenibles, dignas y que ofrezcan la calidad que requiere lo pedagógico, incentivar una cultura que potencie la participación y el compromiso de los ciudadanos con el desarrollo sostenible de la región metropolitana y construir una política pública que garantice condiciones de calidad y equidad de la educación en el Valle de Aburrá a largo plazo (White, Briceño, Arias, Pedraza, Mejía, Lopera, Campuzano, Herrera, Villa, Roll, López, Bedoya, Ramírez, Cadavid, Villa, Cano, Montoya, Toro, Trujillo, Pérez, Osorno y Arango, 2011).

No obstante, sus implicaciones nos llevan a preguntarnos ¿cómo el título de un artículo como éste habla de retos por la sostenibilidad? Esto es, por que en sí misma la sostenibilidad es un desafío de trabajo mancomunado en el que se asocian una serie de interrogantes que vale la pena pensar:

¿Cómo educar para generar el cambio cultural requerido para conjurar las grandes amenazas que se ciernen sobre la
bumanidad? ¿Cómo formar seres humanos que practiquen una ética del bien común, del respeto al otro, de la responsabilidad
social y ambiental? ¿Cómo romper la insensibilidad, la indiferencia, el egoísmo, y motivar actitudes participativas, solidarias y
de liderazgo? ¿Cómo inculcar la conciencia de lo que es debido, el sentido de la mesura, la comprensión del límite o de la
norma que nos regula como seres humanos y sociales? ¿Cómo fomentar el gusto por lo que realmente es bueno y bello para el
hombre? ¿Cómo desarrollar armónicamente las distintas facultades espirituales del ser humano, de modo que los logros
cientificos, técnicos y tecnológicos no se vuelvan contra el hombre mismo y el planeta? En definitiva, ¿cómo transformar nuestra
actual mentalidad "moderna", antropocéntrica, en una mentalidad para la "sostenibilidad? (Posada, en prensa).

Aunque es el planeta el que está manifestando cambios que el hombre no entiende y además de ello le afecta, no son los elementos físicos naturales en donde encontraremos una respuesta a las preguntas enunciadas, sino a la capacidad de decisión y actuación de los seres humanos por mejorar. La sostenibilidad tiene asociada una serie de desafíos por alcanzar en nuestra sociedad actual y debemos asumirla como un aspecto inherente a nuestra forma de vivir y pensar.

En Área Educada, la hemos visto como la oportunidad para analizar y cambiar nuestra forma de ir interpretando y viviendo en el planeta. Esto quiere decir, el excesivo consumo y su desmesurada demanda al comercio, la industria, la moda, como los aspectos que hacen referencia a la intolerancia, la violencia, el aumento de la población mundial a un ritmo exagerado, la pobreza, el desempleo, y el mismo agotamiento de los recursos naturales, se convierte en un tema obligado de la sostenibilidad.

En este artículo, se realiza una investigación sobre la percepción acerca de la sostenibilidad que poseen los participantes de las actividades de Área Educada. Igualmente se realiza una breve descripción de dos actividades que realizamos con público escolar, en las que se encuentran la formación con profesores para introducir la sostenibilidad y el trabajo con estudiantes. No sobra decir, que introducir la sostenibilidad en una región requiere la implicación de otros actores importantes y con los cuales Área Educada trabaja, pero que no son objeto de este trabajo, que son, alcaldes, secretarios de educación y ambiente, empresarios, y demás participantes estratégicos con los que logramos un aporte significativo hacia la sostenibilidad en la región metropolitana y que su desarrollo se encuentra en (White et al, 2011).

Finalmente, ofrecemos recomendaciones, reflexiones y aportes que sirven de base a la comunidad investigativa a incentivar la reflexión, para que inicien y lideren procesos de trasformación social hacia la sostenibilidad.

\section{Hablemos sobre sostenibilidad. ¿La entendemos?}

Existen algunas vertientes sobre cómo se ha abordado la sostenibilidad, una de ellas, la físico natural, otra la conservación y protección de los recursos naturales, de la fauna y de la flora y 
la referida a los aspectos del desarrollo humano (Saura y Hernández, 2008). Para estos autores, la primera tiene que ver con el auge en los años 50 y 70 del cuidado y protección de los recursos, para la segunda y posterior a este periodo hasta finales de los 80 , se vinculan elementos como la defensa y protección del medio ambiente y la relación con la esfera política y económica que se evidencia como la más persistente, y para finales del 2000, la insistencia de la relación de elementos sociales y la responsabilidad de todo el planeta comienza a hacerse más reiterativa. Hay que agregarle a estas definiciones, una característica de los últimos enfoques que ha tenido un gran auge, y es la catastrófica o de emergencia (Vilches y Gil, 2009) incluso difundida por los medios de comunicación y que a nuestro juicio poco contribuye al cambio de percepciones de las personas, y que dejan, restringida la creatividad y las oportunidades de propuestas importantes. Se debe dejar de pensar que ya nada se puede hacer y que las proyecciones aunque desoladoras son las únicas realidades futuras.

En ese sentido, la conciencia sobre la importancia de actuar no está en discusión, pues es la más difundida, sino de cuáles son esas acciones y cuál es el grado de participación y responsabilidad qué tenemos como ciudadanos. Allí, el rol que jugamos como individuos no es claro. Vilches, Gil, Toscano y Macía (2008), ponen en consideración algunos obstáculos que pueden estar impidiendo una implicación de la ciudadanía con la sostenibilidad. Uno de ellos, como se mencionó, es la idea de catastrofe y la imposibilidad de ofrecer respuesta a este problema. Otro obstáculo mencionado es el llamado síndrome de la rana hervida (Gore, 2007) utilizado por el mismo Al Gore como el ejemplo más claro de cómo no nos hemos percatado que el cambio planetario se está presentando por su falta de abordaje contundente y más bien parcial. En Área Educada hemos utilizado un ejemplo para hacer alusión a este obstáculo, Medellín es una de las ciudades que más agua potable y subsidiada por la misma alcaldía ofrece a sus habitantes, las campañas de cuidar el agua porque se está agotando casi no funcionan, debido a que en cualquier parte de la ciudad se abre una llave y el agua fluye por montones, incluso hasta para lavar los coches los domingos. Allí, los cambios ambientales de los que hablan los medios de comunicación no son perceptibles, y la emergencia de la que tanto se habla sobre la crisis del agua no la sienten ninguno de sus habitantes.

A ello, se le agrega la falta de información útil y veraz acerca de las problemáticas contextuales, esto quiere decir que la mayoría de la información que poseemos viene de medios de comunicación o de las llamadas cadenas de e-mails que hacen referencia siempre al oso polar, al panda o a la ballena azul, entre otras, la gran pregunta que nos hacemos es dónde está el conocimiento del contexto; el desconocimiento de lo que nos rodea se convierte en obstáculo. En el caso de Colombia, ser uno de los países más biodiversos nos ha hecho un daño muy grande, pues no alcanzamos ni siquiera a apreciar lo que en abundancia poseemos. Si se desaparece una especie ni si quiera nos daríamos cuenta de lo que ha ocurrido. La sensación de riqueza puede acarrear incluso pobreza mental o sensación de abundancia.

Una creencia generalizada dentro de los mismos profesores, actores fundamentales de este proceso de la sostenibilidad, es que los grandes cambios se dan inicialmente en lo económico y lo político, consideradas como las macroesferas de la sociedad. ¿Qué podría hacer yo?, es una de las preguntas generalizadas de los profesores. Aquí la pirámide del cambio se aprecia desde la dimensiones de poder y se subestima la fuerza del movimiento de masa crítica, de ciudadanos reflexivos y generadores de ideas. El potencial que existe en un aula de clase es un terreno desconocido. Muchas veces no se busca que sea el mismo profesor que genere las grandes ideas, son los niños y las niñas los que tienen mayores ocurrencias, pues sus corazones y sensibilidad están en descubrimiento. Mencionaba una profesora en uno de los tantos talleres que sosteníamos dentro de las actividades que lideramos dentro de Área Educada, la capacidad de enamoramiento que tienen sus niños y la pasión con la cual asumen la labor 
social son desaprovechadas en la tarea de lograr una sostenibilidad en las instituciones escolares.

Si seguimos en la misma línea de lo educativo, la dimensión ambiental ha quedado reducida a proyectos escolares que no salen de actividades de reciclaje o campañas de ahorro de recursos naturales (Mora, 2009). Generalmente los profesores de las áreas de las ciencias lideran los procesos ambientales, pero la pregunta acerca de la participación de los profesores de arte, de valores o de diferentes áreas en estos procesos, queda inconclusa, pues no se le encuentra relación con la sostenibilidad, entre otras cosas, por apreciarse aún la palabra desde la dimensión naturalista del ambiente, lo que en otras palabras se traduce en campañas de no bote papeles al piso, de cierre la llave y apaga el foco, reduciendo al máximo la mirada del ambiente en todas sus dimensiones sociales, científicas, éticas y en últimas humanas . Los currículos de las instituciones educativas no relacionan la sostenibilidad (Alba, Alonso, y Benayas, 2009) por creerse que es un elemento que debe abordarse solo desde las ciencias naturales (Mora, 2009). Igualmente, las estrategias educativas son desconocidas, y falta mayor innovación para acercar a los estudiantes a la sostenibilidad. Aunque, comienzan a surgir propuestas que se descargan de Internet como las que se encuentran en la página web en la sección de Econexión de Área Educada (citada al final del documento) en la que se ofrecen ejemplos y actividades educativas de cómo trabajar la sostenibilidad, así como la página de la OEI (www.oei.es/decada), el número 55 del 2008 de la revista alambique y el volumen 7 del 2010 de la revista Eureka, o trabajos como el de Briceño y Gamboa (2011) una experiencia para evaluar en el ámbito universitario desde todas las áreas y Vilches y Gil (2010) para involucrar actividades en el aula, entre muchas otras más, no resultan suficiente si se desea repercutir en el trabajo educativo para la sostenibilidad, pues son actividades que deben estar contextualizadas dentro del microsistema de la escuela, en la que se pueda trasformar ideas, hábitos y estructuras organizacionales muy arraigadas a lo tradicional (Fernández, 2009). Elementos que sin lugar a dudas, se convierten en retos de la sostenibilidad, estos deben ser asumidos desde una mirada que supere los anteriores obstáculos y propenda por un desarrollo de la persona desde su integralidad y su potencialidad para trasformar su propia realidad (Noguera, 2004).

Es por eso, que abogamos por la concepción de sostenibilidad en la que se trabajan los valores y la toma de decisiones, muy acordes a los aspectos sociales que se describen en la definición del modelo de desarrollo humano de Sura et al, (2008), agregándole, la necesidad de llegar a lo esencial del individuo, pues es la que nos ha permitido creer en que nosotros mismos y nuestras pequeñas voluntades individuales pueden llegar a convertirse en redes y vertientes desencadenantes de cambios. La energía de un niño y niña, la imponencia de un joven y el conocimiento de un profesional pueden desatar trasformaciones.

La sostenibilidad entonces, se refiere a las acciones a favor de la transformación cultural del ser humano a nivel global y de la sociedad, con miras a la superación de las actuales amenazas a la humanidad, (White et al, 2011) que no sólo comprenden la dimensión la conservación y protección de los recursos naturales, de la fauna y de la flora, sino también la social y la económica, y que evidentemente, requiere medidas políticas, tecnológicas, culturales y educativas para hacerle frente, además de las que hemos hecho referencia a las que conciernen al ser, al desarrollo humano (Zabala y García, 2007).

En un ejercicio investigativo, reflexivo y de discusión en el que participaron rectores, profesores, estudiantes, servidores públicos, empresarios, líderes de opinión y expertos, se sustenta el concepto de sostenibilidad como algo integral e inherente a los diversos factores de la vida en sociedad. Estamos hablando que en la elaboración de la sostenibilidad participaron 
alrededor de 1000 personas, gracias al concurso de estos actores se ha llegado a lo siguiente: un ciudadano informado, consiente y más educado será más corresponsable con su entorno y con su propia existencia. Las acciones y decisiones que tome procurarán un mejor desarrollo y estilo de vida, una conciencia ambiental responsable y un pensamiento de respeto por el otro y por sí mismo, es a lo que hemos llamado un ciudadano con visión sostenible.

Así, se enmarca la sostenibilidad en una concepción dinámica que busca sensibilizar y formar al ciudadano, además de desatar procesos que se legitimen en acciones concretas y en la educación como proceso de trasformación para que las personas tengan la oportunidad de cambiar sus vidas, la de sus familias y la de la comunidad, a través de su ejercicio como ciudadanos metropolitanos responsables consigo mismo, con su entorno y con la vida.

La sostenibilidad es una forma de pensar, de sentir y de actuar; la coherencia de cómo logremos una armonía entre estas tres expresiones del ser humano y en la comunicación consigo mismo y con los demás, acorde con un estilo de vida respetuoso y sencillo, lograremos ser un ciudadano sostenible y con una conciencia ambiental responsable.

Como último, resaltamos que la base de una política no necesariamente nace desde los mismo actores políticos, sino que todo aquel actor, llámese estudiante, profesor, rector, director de un centro educativo, secretario de educación, secretario de ambiente, alcalde, gobernador, o político en general, puede verse afectado o motivado sin que necesariamente los primeros cambios se den de manera lineal. Sino que por el contrario un fenómeno educativo e institucional puede llegar a los mismos medios de comunicación y demás colectivos de profesores, promoviendo un diálogo y comunicación efectiva (Briceño y Tafur, 2011). De allí, es que concluimos que en los retos de la sostenibilidad todas las personas tenemos algo que decir, y sobre todo, algo que aportar.

\section{Metodología de la investigación}

No puede considerarse una investigación exclusivamente cuantitativa aun cuando sus resultados son presentados con estadísticas. Esto es porque muchas de las afirmaciones que se mencionaron anteriormente, así como parte de las sugerencias que se hacen en este artículo, vienen de la reflexión conjunta de un equipo interdisciplinario de cerca de 12 personas de todas las profesiones (arquitectos, ingenieros, ambientales, doctores y máster en humanas), con los cuales aportamos una visión cargada de la experiencia de haber ejecutado Área Educada 2009-2011, desarrollado a detalle en la publicación de trasferencia (White et al, 2011) y en la página web del programa (http://www.areaeducada.org/area/).

Sin embargo, en aras de ir organizando la información y de ir respaldando metodológicamente los resultados, se asume que hay un ejercicio deductivo en la medida que nos informamos y compartimos con expertos a la hora de diseñar y proponer las actividades con las que acompañamos profesores y estudiantes, y es inductivo porque parte de los aprendizajes se revierten en el rediseño y perfeccionamiento de cada estrategia de intervención. Los resultados que se presentan se recogen a través de un ejercicio sencillo de percepción donde hacemos una exploración de referentes acerca de qué creen que es la sostenibilidad utilizando las palabras que más asocian al término; se trata de preguntas claras y puntuales y de su cuantificación. La recogida de los datos, se realiza a través de un tiempo de no más de 10 minutos antes de iniciar una actividad, pues buscamos al máximo que las percepciones no se vean afectadas en alguna medida por Área Educada. Generalmente son en aulas de clase donde los estudiantes y profesores están descansados y atentos. 


\section{Momentos}

Aquí presentamos la percepción de dos públicos fundamentales: estudiantes y profesores acerca de la sostenibilidad. Al igual y en los anexos 1 y 2 , se trabajan dos ejemplos para abordar didácticamente la sostenibilidad en el aula. Los momentos que resumen el proceso de la investigación son:

- Formulación de preguntas para explorar referentes de la sostenibilidad contenidas en el anexo 3

- Diseño de actividades para estudiantes y profesores con base en esos referentes

- Resultados

- Discusión

- Elaboración de recomendaciones

- Conclusiones

\section{Diseño de Actividades}

\section{Trabajo con estudiantes}

Aquí se involucran actividades orientadas al trabajo por competencias en las áreas de matemáticas y ciencias, relacionándolas con problemas en sostenibilidad. Este es uno de los primeros ejercicios para involucrar el tema propiamente a las asignaturas. Existe una dificultad de relacionar el currículo con la sostenibilidad pues no es fácil relacionar los problemas que involucran la sostenibilidad con los temas de clase. Aquí las actividades deben buscar analizar problemas ambientales sencillos (ver ejemplo en el Anexo 1).

\section{Trabajo con profesores}

Consiste en talleres dirigidos a los profesores y directivos de las instituciones educativas en los que se trabaja el enfoque de sostenibilidad. Estas jornadas tienen como objetivo promover un espacio de reflexión crítica acerca de cómo incluir este enfoque dentro de la institución a través de sus proyectos educativos e incluso llegando al currículo. Este proceso requiere de un ciclo de actividades formativas que estén encadenas en torno a un objetivo definido con las instituciones. Aquí presentamos el ejemplo de una actividad que se realiza con profesores para identificar las necesidades y acciones fundamentales que el colegio requiere para fortalecer su dimensión en sostenibilidad (ver ejemplo en el Anexo 2).

\section{Participantes}

Dentro de las anteriores actividades descritas, se hizo un trabajo para conocer la percepción de los participantes sobre la sostenibilidad. El papel de Área Educada es ampliar la discusión y reflexión acerca de las implicaciones de la sostenibilidad, tal y como se ha desarrollado en el referente conceptual. Los actores de este estudio fueron en total 360, de los cuales 260 fueron estudiantes principalmente de grados de sexto a noveno, edades entre 12 y 15 años, y 100 profesores de todas las áreas. No se hace discriminación a unas áreas en particular, pues se trabajó con todos los profesores de cinco instituciones educativas distribuidas en toda el Área Metropolitana del Valle de Aburrá, Antioquia Colombia, escogidas intencionalmente, por ser ellas, a las que se les haría adecuaciones de infraestructura, y en otras, construcción de toda la planta física. 


\section{Resultados}

\section{Estudiantes}

Frente a la pregunta qué creemos o entendemos por sostenibilidad, exprésalo en un listado de palabras en una hoja en blanco, los resultados arrojados en la muestra de estudiantes fueron los siguientes:

La mayor frecuencia se presentó en la categoría equilibrio, donde 222 participantes (85\%) asociaron este término a la sostenibilidad, seguida de la categoría proyección a futuro con 186 participantes (71\%), racionalidad con $171(65 \%)$, reciclaje 157 (60\%). La categoría de valores se ubica en una séptima columna con $129(45 \%)$ y se considera que no es un porcentaje bajo.

En la generalidad del gráfico 1, apreciamos un empleo de términos relacionados con un enfoque de desarrollo económico como incremento, abastecimiento, productividad, sustento y racionalidad, así como unas enfocadas al reciclaje, impulsada muy fuertemente por las campañas ambientales que desarrollan las instituciones de una manera reduccionista a la mirada que hacen del ambiente. Los términos como equilibrio y proyección a futuro son los más difundidos dentro de la literatura que habla de desarrollo sostenible y de sostenibilidad, concebidas como un estado natural en el que la sociedad debe vivir relacionada con el ambiente, aparentemente visto como un aspecto idealizado, pues no permite ver las actuales problemáticas del desequilibrio, según los estudiantes todo debe alcanzar un equilibrio per se. Un término importante y de alto interés actual para lo sostenibilidad fue el de valores.

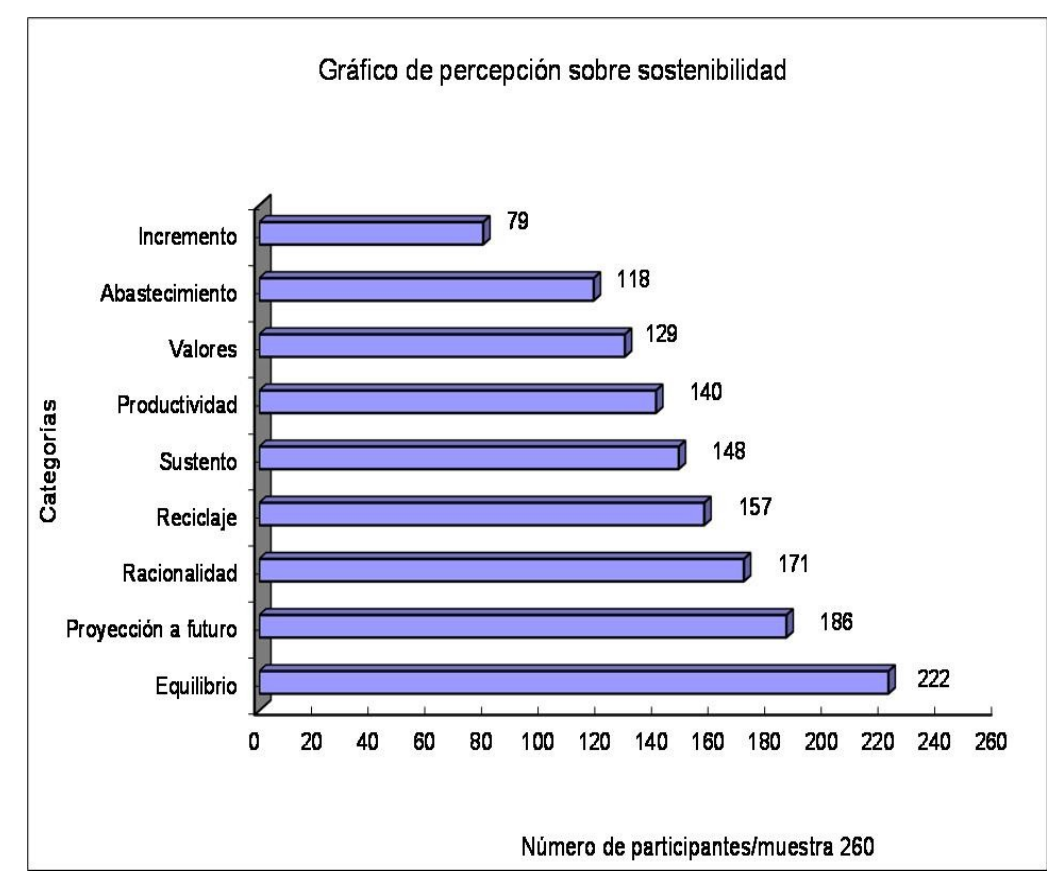

Gráfico 1. Percepción sobre sostenibilidad en estudiantes

En el siguiente gráfico 2, hacemos el listado de palabras que no sumaban más del 5\% del total de respuesta por parte de los estudiantes, pero que valen la pena mostrar, pues se convierten en los términos más importantes a reforzar en un trabajo para la sostenibilidad. Estos están referidos a la ética, a la conciencia, a la práctica, a la igualdad y a la vida, que son los más potentes a la hora de abordar una sostenibilidad tendiente a lo social y el desarrollo humano y al entendimiento de nosotros mismos y nuestra relación con el otro. 


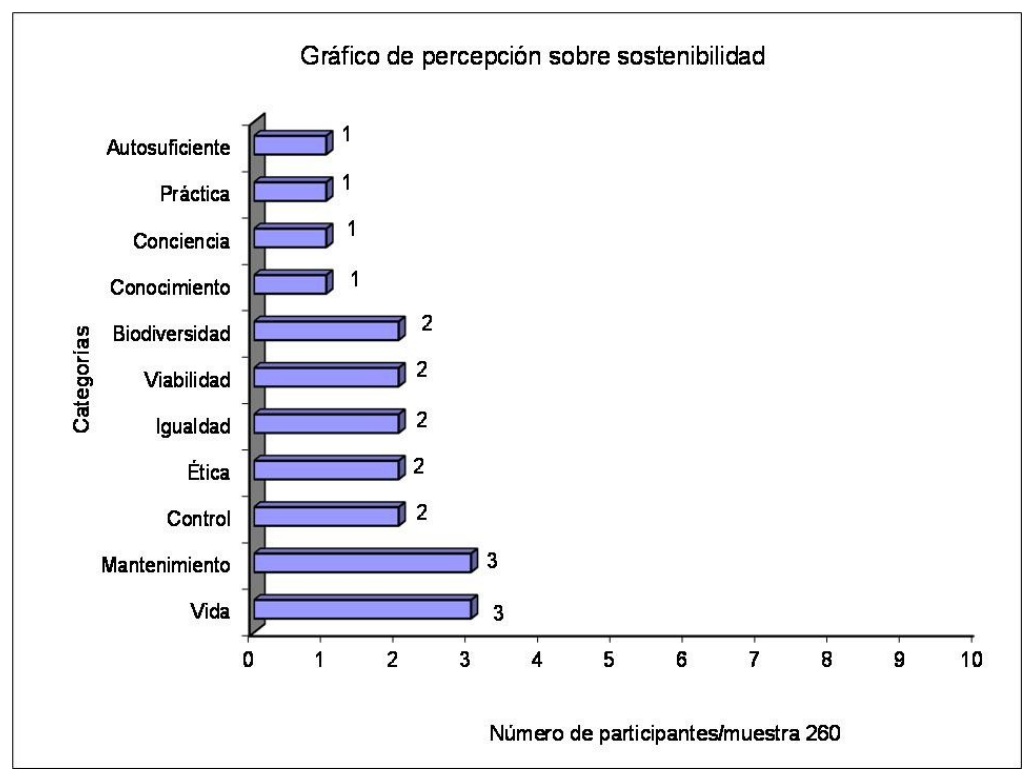

Gráfico 2. Percepción sobre sostenibilidad en estudiantes.

\section{Profesores}

Así como a los estudiantes, se les preguntó a los profesores, en un espacio diferente, qué creen o entienden por la sostenibilidad, expresándolo en un listado de palabras en una hoja en blanco. Los resultados fueron:

La mayor frecuencia la tiene la categoría equilibrio, donde 42 participantes (42\%) asociaron este término a la sostenibilidad, seguida de conservar 34 (34\%) y recursos 29 (29\%) que tuvieron los porcentajes más altos. Continúan las de racionalización, abastecer y responsabilidad.

Llama la atención que salgan expresiones como amor, armonía y pedagogía, aunque en porcentajes muy bajos, pues se ha venido anunciando en el artículo que son palabras de alto interés en el trabajo para la sostenibilidad.

En la generalidad del gráfico 3, se nota que hay una noción tendiente al empleo de términos sobre el conservacionismo y protección de los recursos naturales.

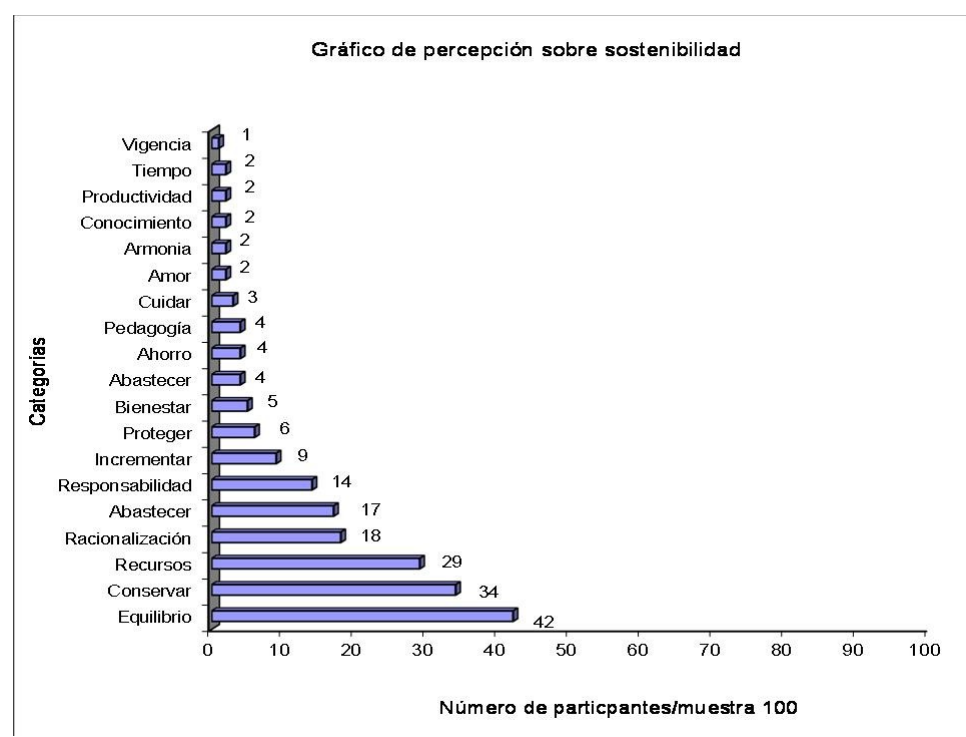

Gráfico 3. Percepción sobre sostenibilidad en profesores. 


\section{Discusión}

En los estudiantes como en los profesores se ve una marcada percepción de la sostenibilidad que tiene que ver con elementos físico naturales y de conservación de los recursos naturales, además del uso de términos como recursos y racionalidad propios de un contexto económico. Hay que mencionar que equilibrio es la categoría más empleada por ambos actores. Esto deja un panorama interesante de analizar, pues hay que ver en qué medida este término no deja de ser una visión ideal de lo que pensamos acerca de cómo debemos mantenernos en relación al ambiente. La justa medida, la noción de no extralimitarnos es una característica de los seres humanos para no trasgredir las mismas normas en las que mantenemos un orden de las cosas. El problema radica en que no sabemos hasta dónde somos conscientes del desequilibrio en el que estamos y de lo que tenemos que hacer para llegar a equilibrarnos de nuevo (Vilches et al 2008), muy ligada a la tesis del antropocentrismo que defiende la idea de que siempre deseamos que todo se vaya acomodando a nuestras necesidades (Noguera, 2004).

La expresión de los valores fue altamente reconocida por los estudiantes como un término que se asocia a la sostenibilidad muy alejada a la manifestada de los profesores. Los estudiantes logran ser más sensibles frente a la visión de la sostenibilidad hacia el desarrollo humano y aspectos sociales.

Es difícil mostrar cuál visión es más fuerte en ambos actores pues se aprecian términos relacionados con lo económico, otros relacionado con lo físico natural y de conservación de los recursos naturales, aunque en gran mayoría aspectos como la ética y el desarrollo humano es más evidente en los estudiantes.

\section{Conclusiones}

Definitivamente hay que atender a los llamamientos internacionales acerca de darle una mirada a la sostenibilidad desde el desarrollo humano y social, superando visiones orientadas únicamente al conservacionismo y proteccionismo de los recursos naturales, que no han respondido a los grandes retos a los que hemos hecho referencia, y que en cambio se siguen manteniendo en las personas, como es el caso de los estudiantes y profesores que participaron de la investigación. Entre otros motivos, porque aún se ubican dentro de un paradigma en la lógica de consumo (Noguera, 2004), en un aprovechamiento ilimitado de los recursos, y una sensación de abundancia que no deja entender la crisis, más allá de las difundidas por los medios de comunicación como el cambio climático.

La noción de desarrollo, incremento y productividad no dejan de ser una visión económica de la situación ambiental, criticada últimamente por los expertos y que en últimas causan confusión, pues no se sabe en qué medida, se puede hablar de un aporte del crecimiento económico a costa de la desmesurada utilización de recursos naturales (Cañal de León y Vilches, 2009). Por el contrario, la dimensión de desarrollo humano es una tendencia que nos invita a revisarnos a nosotros mismos y que se ve asociada al tema de valores, tal y como se refleja en la percepción de los estudiantes, incluso más que en los profesores. Este último elemento, ha venido trabajándose en la literatura referida al tema, en la que se debe repensar la realidad del desarrollo humano como lo describe Programa de las Naciones Unidas para el Desarrollo (PNUD) con (Klugman, 2011), analizando el papel del hombre en la sociedad, su capacidad de trasformación y relacionamiento con los otros, aspectos que fueron defendidos dentro del referente conceptual del articulo y desarrollados por Ârea Educada.

Finalmente, y como elemento confuso, el término de equilibrio es uno de los que mayormente está siendo asociado a la sostenibilidad, esto en ambos actores. Sin embargo, es una palabra ligada al enfoque físico natural, allí la naturaleza ha de estar en una cierta estabilidad entre 
todos los sistemas, siendo un término que muchas veces está cargado de una visión antropocéntrica con la que esperamos deben funcionar las cosas, centrando los problemas en los fenómenos físicos y no en nuestras responsabilidades y acciones directas como individuos.

\section{Recomendaciones}

Para que el trabajo para la sostenibilidad se pueda llevar en contextos educativos se hacen las siguientes recomendaciones:

- Identificar las percepciones que se poseen sobre sostenibilidad en los públicos que se vayan a intervenir.

- Construir de la mano con los participantes del proceso, la visión de la sostenibilidad con base en la reflexión acerca de la sostenibilidad y de sus actuales retos.

- Identificar las problemáticas contextuales de una región, de una localidad o de aquellas específicas de un grupo de personas con las cuales se puedan proponer acciones de mejora o de intervención para solucionarlas. Allí, se aprecia la verdadera sostenibilidad cuando reconocemos nuestra capacidad de acción y poder de trasformación.

- La sostenibilidad no puede verse como un asunto únicamente político y económico, es asunto de todos.

- Los procesos que se realicen con las instituciones educativas deben reconocer los procesos educativos y la historia con la que cuenta una institución.

- La sostenibilidad es una forma de pensar, creer en ella implica vivir en un mejor entorno en el que están implicados los demás actores sociales.

- Los procesos de trasformación son internos, y la mejor forma de llevarlos a los estudiantes es a través del ejemplo.

- El trabajo ambiental implica más allá del mismo entorno natural, sin embargo, hay que llegar más allá de saberlo, debemos interiorizarlos con acciones concretas.

- No es fácil reconocer cómo abordar el ambiente desde su multiplicidad de saberes, pero hay que esforzarse cada día por aprender cada vez más cómo lograrlo

- Trabajar en la mente y emociones de los niños, niñas y jóvenes es el mejor elemento de trasformación social.

- Hay que promover mayor claridad a la comunidad de educadores evitándoles confusiones terminológicas, pues incluso se ha llegado a poner como antagónico la sostenibilidad con la educación ambiental.

\section{Referencias bibliográficas}

Alba, D., Alonso, I. y Benayas, J. (2009). Universidad y sostenibilidad: más allá del currículo. Cuadernos de pedagogia: ejemplar dedicado a: educación ambiental para la sostenibilidad, 392, 36-38

Briceño, J. y Gamboa, M. (2011). El portafolio: una estrategia para la enseñanza de las ciencias. Experiencia llevada en una universidad colombiana. Revista Eureka Enseñanza y Divulgación de las Ciencias, 8, 1, 84-92. En línea en: http://reuredc.uca.es/index.php /tavira/article/view/63

Briceño, J. y Tafur, M. (2011). Caracterización del diálogo guía-estudiante en un Museo Interactivo de Ciencia y Tecnología de Bogotá (Colombia). Revista Electrónica Enseñanz̧a 
de las Ciencias, 10, 2, 289-177. En línea en: http://reec.uvigo.es /volumenes/volumen10/ART4_VOL10_N2.pdf

Cañal, P. y Vilches, A. (2009). El rechazo del desarrollo sostenible: ¿una crítica justificada? Enseñanza de las Ciencias, Número Extra VIII Congreso Internacional sobre Investigación en Didáctica de las Ciencias, Barcelona, 677-680. En línea en: http://ensciencias.uab.es/congreso09/numeroextra/art-677-680.pdf

Fernández, M. (2009). Resumen de estrategias hacia la sostenibilidad. Revista Cuadernos de pedagogía, 392, 30-33.

Gore, A. (2007). Una verdad incómoda. Ed. Gedisa. Barcelona, España.

Klugman, J. (2011). Informe sobre desarrollo bumano 2011. Sostenibilidad y equidad: Un mejor futuro para todos. Ed. Programa de las Naciones Unidas para el Desarrollo (PNUD). Nueva York, E.U. En línea en: http://hdr.undp.org/en/media/HDR_2011_ES_ Complete.pdf

Mora, W. (2009). Crisis y educación ambiental para la sustentabilidad: retos a los procesos formativos del profesorado. Revista Tecné, Episteme y Didaxis: TED No. Extraordinario, 54-76. En línea en: http://www.pedagogica.edu.co/revistas/ojs/index.php/TED /article/viewDownloadInterstitial/175/150

Noguera, P. (2004). El reencantamiento del mundo. Ed. Programa de las Naciones Unidas para el Medio Ambiente - PNUMA - Oficina Regional para América Latina y el Caribe y Universidad Nacional de Colombia. IDEA. Colombia.

Posada, M. (En prensa). "Qué entendemos por sostenibilidad” Programa Área Educada: Educación con calidad para la sostenibilidad. Ed. Centro de Ciencia y Tecnología de Antioquia y Área Metropolitana del Valle de Aburrá.

Saura, P. y Hernández, M. (2008). La evolución del concepto de sostenibilidad y su incidencia en la educación ambiental. Revista Teoría Educación, 20, 179-204. En línea en: http://campus.usal.es/ revistas_trabajo/index.php/1130-3743/article/viewFile/989/ 1087

Vilches, A., Gil, D., Toscano, J. y Macías, O. (2008). Obstáculos que pueden estar impidiendo la implicación de la ciudadanía y, en particular, de los educadores, en la construcción de un futuro sostenible. Formas de superarlos. Revista CTS, 11, 4, 139-162.

Vilches, A., Gil, D. (2009). Una situación de emergencia planetaria, a la que debemos y "podemos" hacer frente. Revista de Educación, N extra 1, 101-122. En línea en: http://www.oei.es/decada/re2009_05.pdf

Vilches, A., Gil, D. (2010). ¿Cómo puede contribuir la educación a la construcción de u futuro sostenible? Revista eureka Enseñanza y Divulgación de las Ciencias, $N$ extraordinario, 291-315.

White, B., Briceño, J., Arias, A., Pedraza, M., Mejía, D., Lopera, A., Campuzano, C., Herrera, C., Villa, A., Roll, D., López, A., Bedoya, M., Ramírez, J., Cadavid, C., Villa, A., Cano, A., Montoya, M., Toro, G., Trujillo, J., Pérez, Z., Osorno, C., y Arango, A. (2011). Publicación de trasferencia, Modelo Area Educada: educación con calidad para la sostenibilidad., Ed. Centro de Ciencia Medellín, Colombia.

Zabala, I. y García, M. (2007). La Educación del Siglo XXI de acuerdo a la perspectiva del paradigma ecológico: Una Alternativa para la sostenibilidad. Revista de Investigación, 68, $33,233-250$. 


\section{Páginas web relacionadas}

Econexión (ene.11, 2012). En línea en: http://www.reddebibliotecas.org.co /comunidadambiental /Paginas/default.aspx 
Anexo 1. Actividad: entendiendo el ciclo de una inadecuada acción con ejemplos fáciles de comprender

Objetivo: Concienciar acerca del uso irresponsable que hacemos del recurso hídrico, a través de operaciones básicas de matemáticas

Desarrollo de la actividad a través de una $\quad$ ¿Cuánta agua gastamos?

pregunta

Sabias qué...

Un litro de agua se puede

representar en la siguiente botella

Al día puedes estar consumiendo 100 botellas

$\mathrm{Al}$ mes estás consumiendo 3000 mil botellas

En un año 36000 mil botellas

Sabias qué

Si fueran 5 en una familia, podrías quitarles la sed a todos los habitantes del municipio de Envigado (Antioquia- Colombia) por un día y que son aproximadamente 180.000

Cuando han entendido el ejemplo, y se realiza el análisis, lanzamos más preguntas:

Te imaginas cuánta agua consumiríamos dependiendo de

Cuántas familias hay en tu barrio

Cuántas familias hay en tu ciudad

Cuántas familias hay en tu país

Has un cálculo aproximado acerca de cuánta agua gastamos por día en el país, si aproximadamente hay en Colombia 42.888.594 habitantes

Con esa agua ¿a cuántas personas les darías de beber en un día?

Recomendaciones

La actividad resulta mucho más potente cuando involucramos ejemplos utilizando al menos 20 botellas en una mesa, seguido de ejercicios mentales para relacionar el número de botellas que se requieren para llenar un estadio de fútbol. Muchas veces los estudiantes no dimensionan la magnitud de las cifras grandes a menos que se le asocie la cantidad de botellas con una unidad de medida que puedan asociar, en este caso, el municipio de Envigado es un ejemplo que le permite a los estudiantes dimensionar que estamos hablando de una cantidad muy grande, producida por una sola familia al año. 


\section{Anexo 2. Actividad: elaborando planes de acción con profesores}

\section{Introducción de la actividad}

Los planes de acción que se realizan en conjunto con las instituciones educativas deben entenderse como el conjunto de actividades o acciones dispuestas y ordenadas en una perspectiva de cumplimiento de objetivos y un enfoque sobre la sostenibilidad, acordes con un consenso entre los profesores y directivos de cada institución y contextualizados a un horizonte institucional enmarcados en sus Proyectos Educativos Institucionales (PEI) y en sus Proyectos Ambientales Escolares (PRAE). Cabe resaltar que los planes de acción superan la mirada única de actividades sueltas y se relacionan con una visión planificada.

Se recomienda trabajar en un taller anterior a este, la siguiente pregunta: ¿qué se entiende por sostenibilidad? Tal y como se desarrolló en la presente investigación, esto es, porque es necesario ir definiendo con los profesores el enfoque con el cuál desean trabajar su plan de acción, superando a medida que se van exteriorizando las percepciones, enfoques centrados únicamente en el uso racional de los recursos naturales a unas más acordes a las desarrolladas en el presente artículo, denominadas sociales y humanas.

Es así, que los planes de acción son herramientas que permiten ver de manera organizada unas intenciones de trabajo, y para organizarlas utilizamos tabla 1 en la que se ordena en columnas y filas, las actividades, los objetivos y la proyección de las mismas en un intervalo de tiempo.

La visión y misión serán las mismas que las instituciones hayan definido en sus PEI y PRAE. No obstante este ejercicio les permite ir reevaluando algunos aspectos definidos en sus propios documentos institucionales que se van incorporando a la tabla y la concepción de sostenibilidad que han venido construyendo.

Finalmente, el éxito de los planes de acción está en que casi siempre las instituciones afirman que requieren fortalecimientos, pero aquí todas estas acciones están centradas en un propósito mancomunado de todos los profesores, en una delimitación interesante de sus visiones y misiones tanto del PEI como del PRAE, y en un enfoque sobre la sostenibilidad a la que apostarán todos los profesores.

\section{Desarrollo del taller que nos llevó a los planes de acción}

\section{Finalidad}

Durante una jornada de tres horas, se devuelven algunos elementos claves de las percepciones acerca del término sostenibilidad, obtenidos previamente con los profesores. Igualmente, se recomienda que los planes de acción tengan en cuenta diagnósticos institucionales donde se pueda establecer en alguna medida las necesidades de los profesores frente a la educación para la sostenibilidad, con el fin de que se reconozcan los elementos más críticos que requieren fortalecimiento. De esta manera se hace una relación directa entre las necesidades de las I.E. frente a las acciones.

\section{Metodología}

Durante la primera hora se realiza una breve presentación de las líneas concretas donde se encuentran mayores debilidades en las instituciones basadas en el diagnóstico y los resultados de las percepciones sobre la sostenibilidad. De esta forma, se detalla el grado de aceptación y se escuchan aportes y comentarios.

Una vez se valida con los profesores este ejercicio, se pasa a trabajar en las siguientes dos horas en el plan de acción, a partir de un ejercicio en el que se relacionen esas necesidades con las acciones necesarias para desarrollar o fortalecer los procesos institucionales. Así logramos 
que los planes de acción involucren toda la reflexión realizada sobre la sostenibilidad y los diagnósticos, y aporten de manera más precisa a un fortalecimiento concreto y articulado a sus necesidades.

Los planes de acción son una estrategia concreta donde se vislumbra un objetivo y acciones entorno a un enfoque de la sostenibilidad.

La tabla 1 que sistematizará ese proceso es la siguiente:

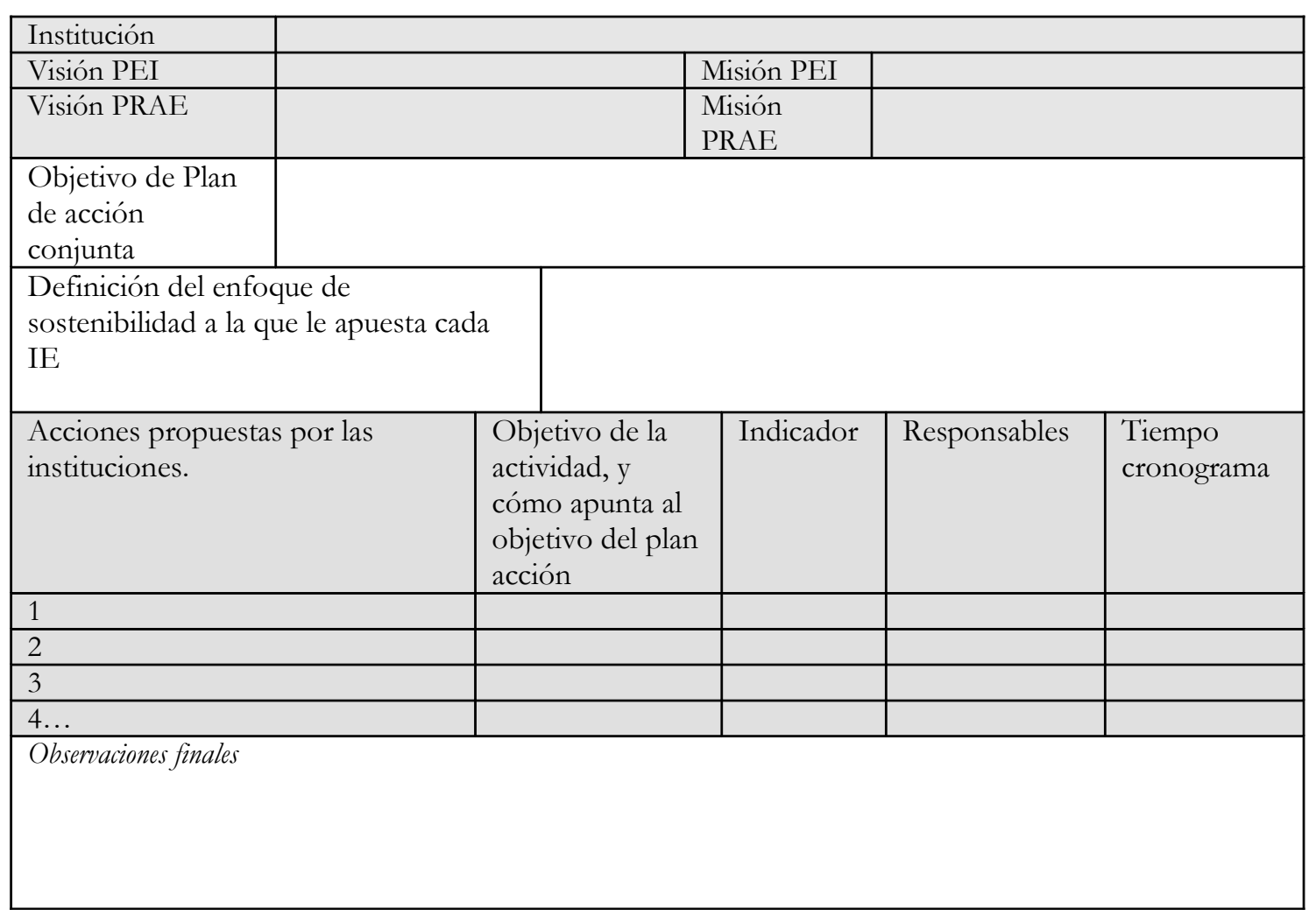


Anexo 3. Preguntas para indagar las percepciones de profesores y estudiantes sobre la sostenibilidad
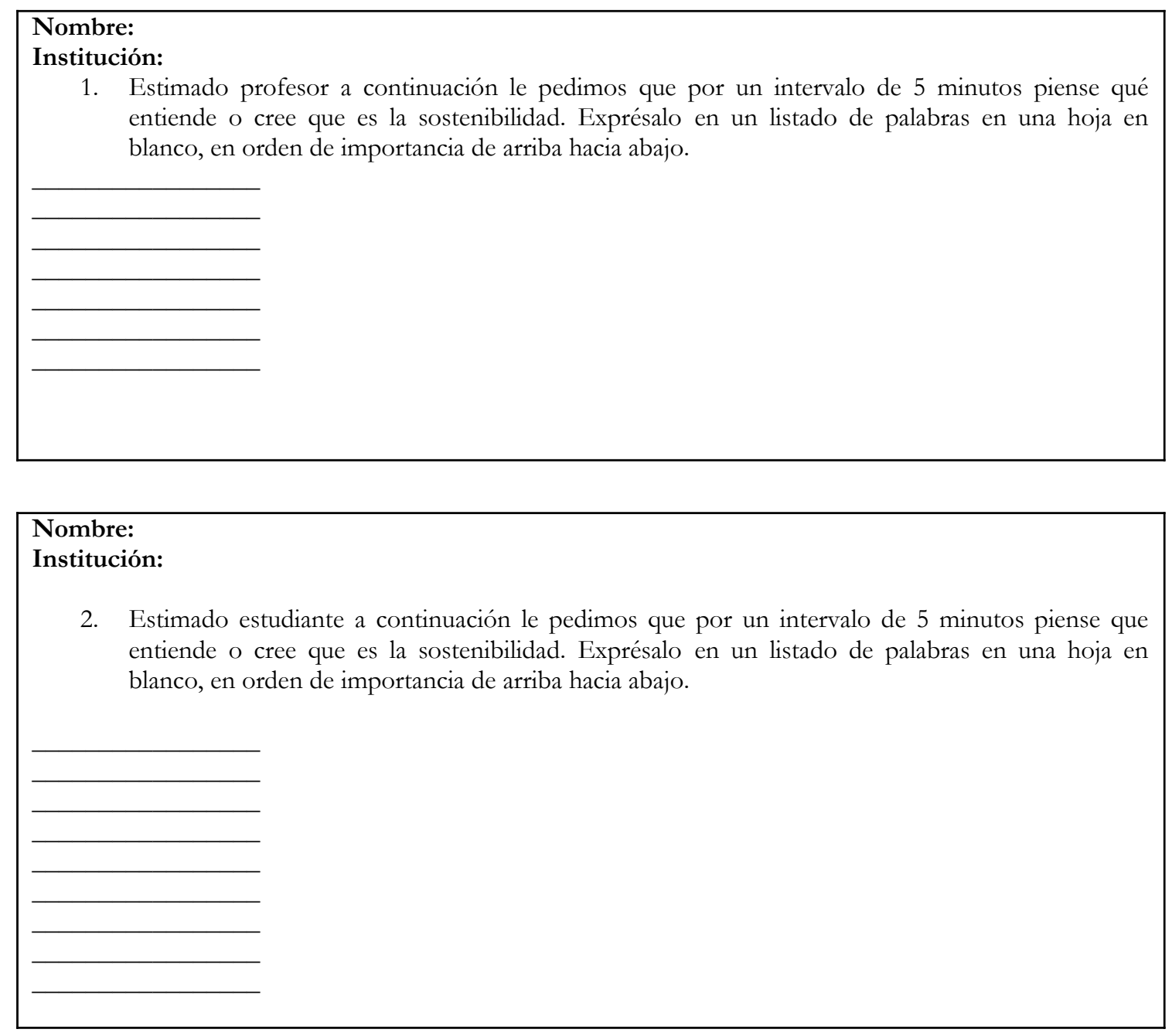